\title{
Simple analytical models for the calculation of the electric field radiated by the base station antenna
}

\author{
Marin Galić(1), Dragan Poljak(2), Vicko Dorić( $(2)$ \\ (1) Centar za mjerenja u okolišu d.o.o. (Environmental Measurement Center LTD), University of Split, FESB \\ e-mail:mgalic@cmo.hr \\ (2) University of Split, FESB \\ e-mails:dpoljak@fesb.hr; vdoric@fesb.hr
}

\section{SUMMARY}

This paper outlines four different models for the assessment of the electric field radiated by the base station antenna system, thus featuring the Free Space Model (FS), Perfect Ground Model (PG), Fresnel Reflection Coefficient Model (RC) and Modified Image Theory Model (MIT). The results obtained with these approaches are compared to the results computed via Numerical Electromagnetics Code (NEC). Several variables have been varied to examine the accuracy of each calculation model. The calculations have been undertaken for the far field only. As many European countries perform the field assessment using the FS model, the final goal of this work is to come up with the most convenient model for the field calculation.

KEY WORDS: $\quad$ electric field strength estimation; free space; perfect ground; modified image theory method; Fresnel equation; far field area.

\section{INTRODUCTION}

The presence of high frequency (HF) electromagnetic fields in the environment due to base station antenna systems has caused a continuous public concern regarding possible radiation hazard. Namely, at HF exposures the human body may absorb a significant amount of the radiated energy, as the dimensions of organs are comparable to the wavelength of the incident field and the dominant biological effect is the tissue heating [1-2]. The problem is by itself twofold including: first, the assessment of the external field (incident field dosimetry), and second, the rate of power deposition in tissue due to the exposure to HF radiation and the related temperature distribution within the body has to be determined.

This paper is the sequel to two previous articles of the authors [3-4] and deals with the simple analytical relations for the incident field dosimetry based on the different schemes of ray tracing algorithm (arising from the geometrical optics approach $[5,6]$ implementation. Four 
different models for the assessment of the field radiated by the base station antenna are presented; the antenna insulated in Free Space (FS), the antenna above Perfect Ground (PG) and the antenna above a lossy half space. The half-space model uses the reflection coefficient stemming from the Snell's law and Fresnel's equation [7-8] but also from the MIT approximation [9-10]. The paper is organized as follows: Mathematical Model is presented in Section II, and it is followed by a number of illustrative computational results. Finally, some concluding remarks are given. The results obtained by three different models are compared to the results computed via Numerical Electromagnetics Code (NEC) [11]. Several variables have been varied to examine the accuracy of a given approximation. All calculations have been carried out for the far field zone.

\section{MATHEMATICAL MODELS FOR INCIDENT DOSIMETRY OF THE BASE STATION ANTENNAS}

\subsection{FREE SPACE APPROXIMATION}

The Free Space approximation neglects effects due to reflections and scattering, thus taking into account only the incident field, Figure 1.

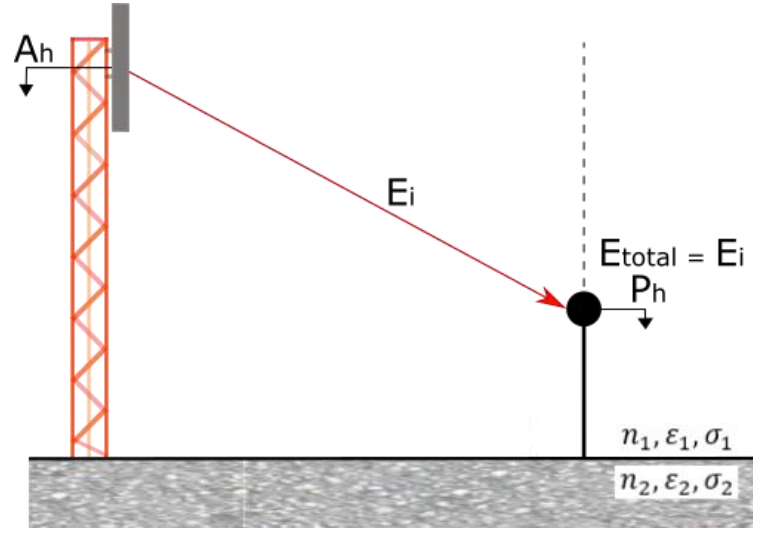

Fig. 1 Free Space Approximation

As documented in [12] the electric field at some point in free space in the far-field zone is given by:

$$
E_{\text {total }}=E_{i}=\frac{\sqrt{30 * N * P * 10^{\frac{G i}{10}}}}{d i}(\mathrm{~V} / \mathrm{m})
$$

where $N$ is number of channels, $P$ is radiated power $(W), d_{i}$ is the distance between the antenna and the calculation point $(m)$ and $G_{i}$ is antenna gain of incident field $(d B)$.

Formula (1) gives the absolute value of the total electric field according to the assumptions given at the beginning of the section. 


\subsection{PERFECT GROUND APPROXIMATION}

A simple way to take into consideration the effect of the reflected wave on total electric field is to assume the ground to be perfectly conducting, Figure 2 .

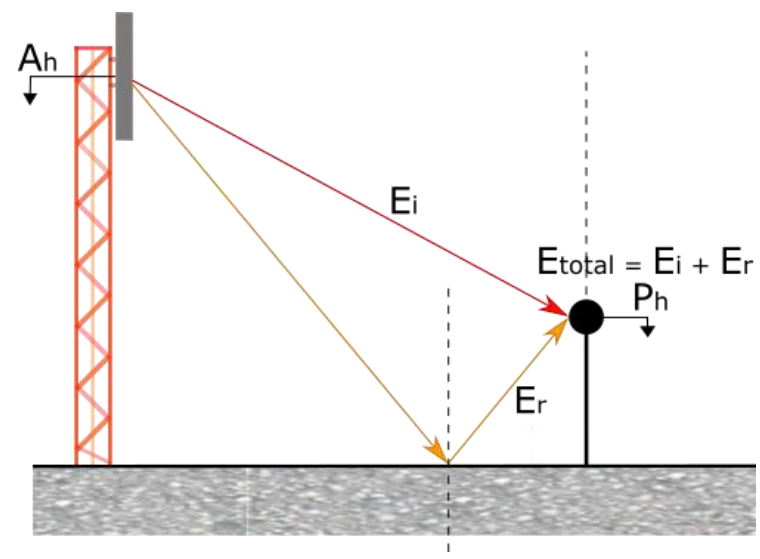

Fig. 2 Perfect Ground Approximation

Therefore, the total electric field is:

$$
\overrightarrow{E_{\text {total }}}=\overrightarrow{E_{i}}+\overrightarrow{E_{r_{-} P G}}(\mathrm{~V} / \mathrm{m})
$$

where:

$$
E_{r_{-} P G}=\frac{\sqrt{30 * N * P * 10^{\frac{G r}{10}}}}{d r}(\mathrm{~V} / \mathrm{m})
$$

with $d_{r}$ being the distance that wave travels from the antenna to the calculation point and $G_{r}$ is the antenna gain.

\subsection{HALF-SPACE MODEL FEATURING FRESNEL'S APPROACH}

The total field composed from both, incident and reflected field, can also be calculated using RC or MIT method, respectively, Figure 3.

Reflection coefficient arising from RC approach is given by:

$$
\Gamma_{\text {Fresnel }}=\frac{Z_{1} * \cos \alpha-Z_{2} * \cos \beta}{Z_{1} * \cos \alpha+Z_{2} * \cos \beta}
$$

where $Z_{1}$ is the free space impedance $(377 \Omega)$ and $Z_{2}$ is given by:

$$
Z_{2}=\sqrt{\frac{j \mu_{0} \cdot \omega}{\sigma_{2}+j \varepsilon_{0} \varepsilon_{2} \cdot \omega}}
$$

The angle $\beta$ stems from Snell's law, as follows:

$$
n_{1} * \sin \alpha=n_{2} * \sin \beta
$$

where $n_{2}$ is given by:

$$
n_{2}=\sqrt{\varepsilon_{2}}
$$


Thus, the reflected electric field is:

$$
\overrightarrow{E_{r_{-} \text {Fresnel }}}=\Gamma_{\text {Fresnel }} * \overrightarrow{E_{r_{-} P G}}(\mathrm{~V} / \mathrm{m})
$$

and the total electric field strength is:

$$
\overrightarrow{E_{\text {total }}}=\overrightarrow{E_{i}}+\overrightarrow{E_{r_{-} \text {Fresnel }}}(\mathrm{V} / \mathrm{m})
$$

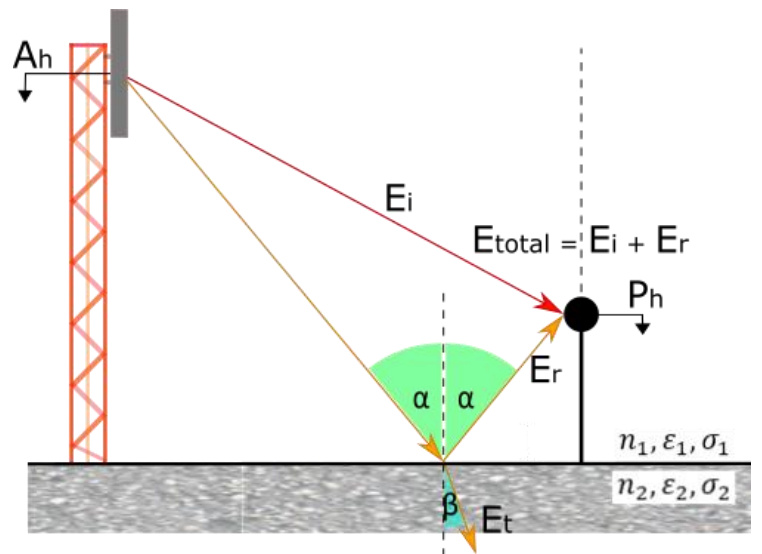

Fig. 3 Antenna above a lossy half space

\subsection{HALF-SPACE MODEL FEATURING MIT APPROACH}

Reflection coefficient arising from MIT approach is given by:

$$
\Gamma_{M I T}=\frac{\varepsilon_{e f f}-\varepsilon_{0}}{\varepsilon_{e f f}+\varepsilon_{0}}
$$

where:

$$
\varepsilon_{e f f}=\varepsilon_{r} \varepsilon_{0}+\frac{\sigma}{j \omega}(F / m)
$$

Thus, the reflected electric field is:

$$
\overrightarrow{E_{r_{-} M I T}}=\Gamma_{M I T} * \overrightarrow{E_{r_{-} P G}}(\mathrm{~V} / \mathrm{m})
$$

Therefore, the total electric field is:

$$
\overrightarrow{E_{\text {total }}}=\overrightarrow{E_{i}}+\overrightarrow{E_{r_{-} M I T}}(\mathrm{~V} / \mathrm{m})
$$

\section{THE RESULTS}

\subsection{ANTENNA CONFIGURATION}

Antenna configuration of interest is $2 \mathrm{~m}$ long and consists of 8 dipoles and metal grid with the total radiated power of $100 \mathrm{~W}$. The operating frequency is $936.8 \mathrm{MHz}$. Simulations are carried out by varying specific ground conductivity $\sigma_{2}$, relative permittivity $\varepsilon_{2}$ and the antenna height $A_{h}$. Values of these variables used in simulations are shown in Table 1 . The theoretical antenna pattern is shown in Figure 4. 
Table 1 Values of variables

\begin{tabular}{|c|c|c|c|c|c|c|}
\hline variable & $\mathbf{1}$ & $\mathbf{2}$ & $\mathbf{3}$ & $\mathbf{4}$ & $\mathbf{5}$ & $\mathbf{6}$ \\
\hline$\sigma_{2}(\mathrm{~S} / \mathrm{m})$ & $10^{-3}$ & 0.01 & 0.1 & 10 & $10^{3}$ & $10^{6}$ \\
\hline$\varepsilon_{2}$ & 10 & 40 & 80 & 110 & 150 & - \\
\hline$A_{h}(\mathrm{~m})$ & 10 & 20 & 30 & 40 & 50 & - \\
\hline
\end{tabular}

Other variables used in simulations are set to be constant, i.e.: radiated power $(P=100 \mathrm{~W})$, channel number $(N=1)$, calculation point height $\left(P_{h}=2 \mathrm{~m}\right)$.

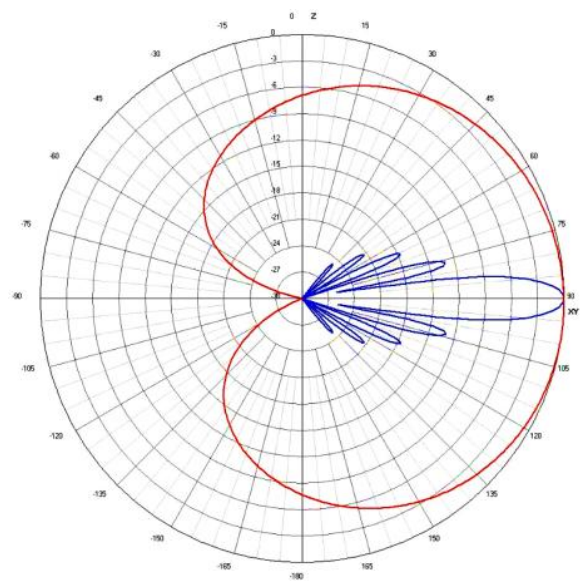

Fig. 4 Perfect Ground Approximation

As already stated, all calculations have been carried out for the far field zone [13]:

$$
d \geq 2 * D^{2} / \lambda \quad(V / m)
$$

where $d$ is horizontal distance from the antenna, $D$ is the largest antenna dimension and $\lambda$ is wavelength. Calculating with $D=2 \mathrm{~m}$ and $\lambda=0.32 \mathrm{~m}$, far field area is related to distances from $25 \mathrm{~m}$. Therefore, the electric field is calculated for distances between $25 \mathrm{~m}$ to $400 \mathrm{~m}$ away from antenna with the step of $1 \mathrm{~m}$ (total 376 data per calculation).

\subsection{VARIATIONS OF THE GROUND CONDUCTIVITY}

The differences in obtained results among the proposed methods with respect to the conductivity variations are presented in Tables 2 and 3, and Figures 5 and 6.

Table 2 The highest absolute differences between each method and NEC simulation in $\mathrm{mV} / \mathrm{m}$

\begin{tabular}{|c|c|c|c|c|}
\hline $\boldsymbol{\sigma}_{2}(\boldsymbol{S} / \boldsymbol{m})$ & $\boldsymbol{F S}-\mathbf{N E C}$ & $\boldsymbol{P G}-\boldsymbol{N E C}$ & $\boldsymbol{R C}-\boldsymbol{N E C}$ & $\boldsymbol{M I T}-\boldsymbol{N E C}$ \\
\hline \multirow{2}{*}{0.001} & 70,87 & 198,11 & 51,32 & 174,27 \\
\cline { 2 - 5 } & $36,99 \%$ & $220,19 \%$ & $53,48 \%$ & $193,69 \%$ \\
\hline \multirow{2}{*}{0.01} & 70,99 & 198,07 & 51,42 & 174,25 \\
\cline { 2 - 5 } & $37,02 \%$ & $220,06 \%$ & $53,60 \%$ & $193,21 \%$ \\
\hline \multirow{2}{*}{0.1} & 72,00 & 197,59 & 52,91 & 174,51 \\
\cline { 2 - 5 } & $37,36 \%$ & $217,61 \%$ & $55,16 \%$ & $192,43 \%$ \\
\hline \multirow{2}{*}{10} & 94,74 & 110,89 & 128,75 & 110,42 \\
\cline { 2 - 5 } & $42,37 \%$ & $66,50 \%$ & $89,87 \%$ & $66,22 \%$ \\
\hline \multirow{2}{*}{1000} & 130,55 & 124,54 & 126,98 & 124,54 \\
\cline { 2 - 5 } & $49,42 \%$ & $77,61 \%$ & $79,13 \%$ & $77,61 \%$ \\
\hline \multirow{2}{*}{$10^{6}$} & 140,06 & 126,50 & 126,58 & 126,50 \\
\cline { 2 - 5 } & $47,12 \%$ & $77,88 \%$ & $77,93 \%$ & $77,88 \%$ \\
\hline
\end{tabular}


Table 3 Percentage of results for the given method within distortion up to $25 \%$ of NEC results

\begin{tabular}{|c|c|c|c|c|}
\hline $\boldsymbol{\sigma}_{\boldsymbol{2}}(\boldsymbol{S} / \boldsymbol{m})$ & $\boldsymbol{F S}-\boldsymbol{N E C}$ & $\boldsymbol{P G}-\boldsymbol{N E C}$ & $\boldsymbol{R C}$ - $\boldsymbol{N E C}$ & $\boldsymbol{M I T}$-NEC \\
\hline 0.001 & $63 \%$ & $29 \%$ & $93 \%$ & $32 \%$ \\
\hline 0.01 & $62 \%$ & $29 \%$ & $93 \%$ & $32 \%$ \\
\hline 0.1 & $62 \%$ & $30 \%$ & $93 \%$ & $32 \%$ \\
\hline 10 & $44 \%$ & $48 \%$ & $93 \%$ & $48 \%$ \\
\hline 1000 & $39 \%$ & $93 \%$ & $93 \%$ & $93 \%$ \\
\hline $10^{6}$ & $37 \%$ & $93 \%$ & $93 \%$ & $93 \%$ \\
\hline
\end{tabular}

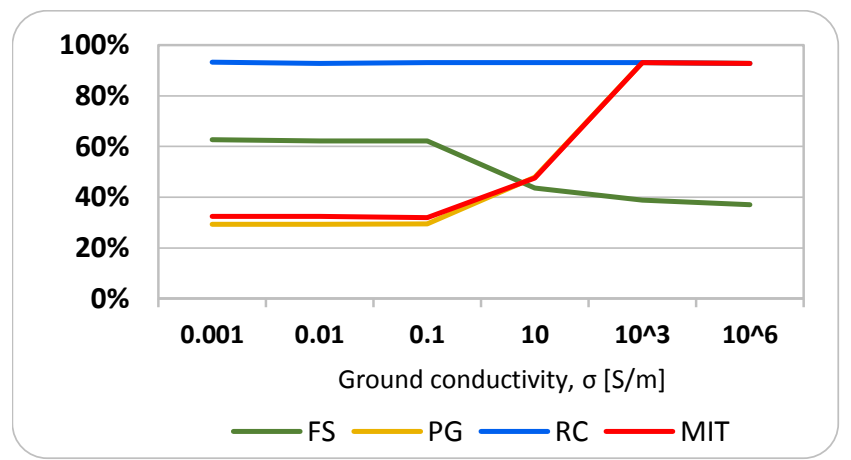

Fig. 5 Percentage of results for the given method within distortion up to $25 \%$ of NEC results for different $\sigma_{2}$

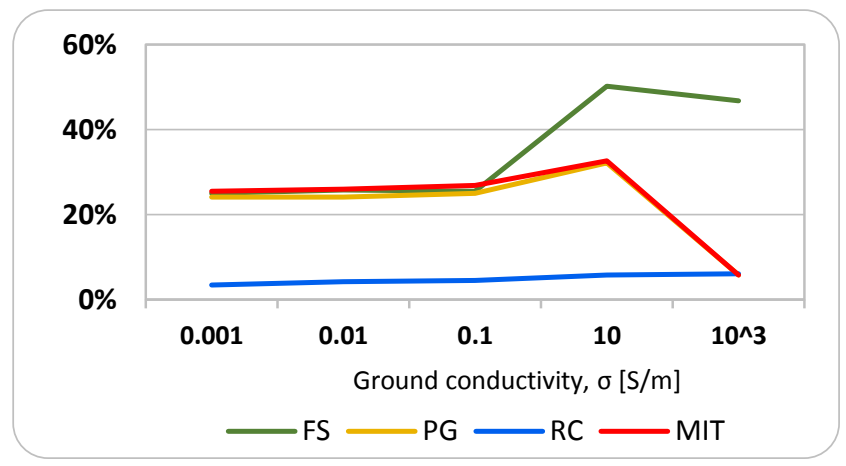

Fig. 6 Percentage of results for the given method with distortion between 25\% and 50\% of NEC results for different $\sigma_{2}$

The increase of ground conductivity has negligible influence on total electric field strength. The RC approximation appears to be optimal while FS method, on the other hand, provides more accurate results than PG or MIT method for conductivity values less than $10 \mathrm{~S} / \mathrm{m}$. Increasing the ground conductivity to $1000 \mathrm{~S} / \mathrm{m}$ or even $10^{6} \mathrm{~S} / \mathrm{m}$, PG, MIT and RC models provides similar results, while FS method fails to ensure valid results.

\subsection{VARIATIONS OF THE RELATIVE PERMITTIVITY}

The differences in simulated results among the proposed methods for the permittivity variations are presented in Tables 4 and 5 and Figures 7 and 8. 
Table 4 The highest absolute differences between each method and NEC simulation in $\mathrm{mV} / \mathrm{m}$

\begin{tabular}{|c|c|c|c|c|}
\hline \multirow{2}{*}{$\boldsymbol{\varepsilon}_{\boldsymbol{2}}$} & $\boldsymbol{F S}$ - $N E \boldsymbol{C}$ & $\boldsymbol{P G}-\mathbf{N E C}$ & $\boldsymbol{R C}-\boldsymbol{N E C}$ & $\boldsymbol{M I T}-\boldsymbol{N E C}$ \\
\hline \multirow{2}{*}{10} & 70,99 & 198,07 & 51,42 & 174,25 \\
\cline { 2 - 5 } & $37,02 \%$ & $220,06 \%$ & $53,60 \%$ & $193,21 \%$ \\
\hline \multirow{2}{*}{40} & 52,73 & 163,15 & 100,26 & 156,76 \\
\cline { 2 - 5 } & $32,13 \%$ & $130,85 \%$ & $84,07 \%$ & $125,72 \%$ \\
\hline \multirow{2}{*}{80} & 66,64 & 141,63 & 115,95 & 138,39 \\
\cline { 2 - 5 } & $63,57 \%$ & $97,27 \%$ & $89,70 \%$ & $95,05 \%$ \\
\hline \multirow{2}{*}{110} & 72,46 & 131,26 & 120,00 & 128,90 \\
\cline { 2 - 5 } & $65,49 \%$ & $84,16 \%$ & $90,04 \%$ & $82,65 \%$ \\
\hline \multirow{2}{*}{150} & 77,66 & 121,05 & 122,41 & 119,32 \\
\cline { 2 - 5 } & $67,04 \%$ & $73,01 \%$ & $89,46 \%$ & $71,96 \%$ \\
\hline
\end{tabular}

Table 5 Percentage of results for the given method within distortion up to 25\% of NEC results

\begin{tabular}{|c|c|c|c|c|}
\hline $\boldsymbol{\varepsilon}_{2}$ & $\boldsymbol{F S}$ - $\boldsymbol{N E C}$ & $\boldsymbol{P G}$-NEC & $\boldsymbol{R C}$-NEC & $\boldsymbol{M I T}$-NEC \\
\hline 10 & $62 \%$ & $29 \%$ & $93 \%$ & $32 \%$ \\
\hline 40 & $86 \%$ & $30 \%$ & $94 \%$ & $32 \%$ \\
\hline 80 & $88 \%$ & $32 \%$ & $93 \%$ & $31 \%$ \\
\hline 110 & $88 \%$ & $32 \%$ & $93 \%$ & $34 \%$ \\
\hline 150 & $86 \%$ & $35 \%$ & $93 \%$ & $36 \%$ \\
\hline
\end{tabular}

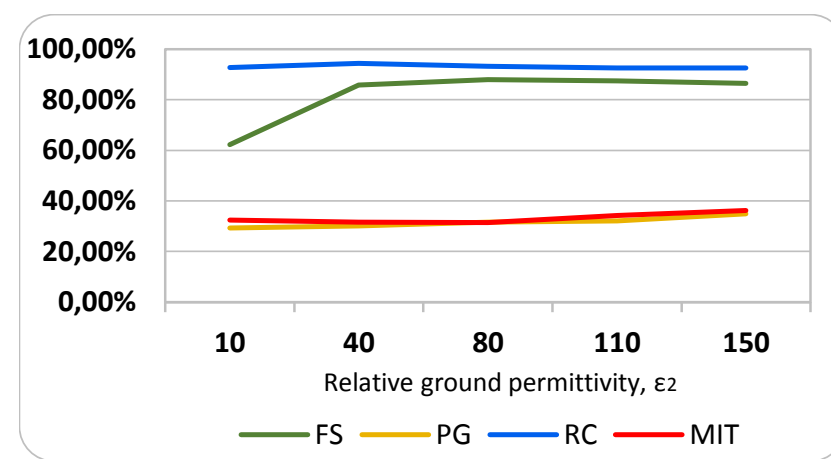

Fig. 7 Percentage of results for the given method within distortion up to 25\% of NEC results for different $\varepsilon_{2}$

RC method is considered to be the most accurate approach with approximately $93 \%$ of data with distortion up to $25 \%$ of NEC results. FS method provides better results as relative permittivity values increase above 10. PG and MIT methods show similar results but also suffer from the low accuracy with maximum $36 \%$ of data with distortion up to $25 \%$ of NEC results.

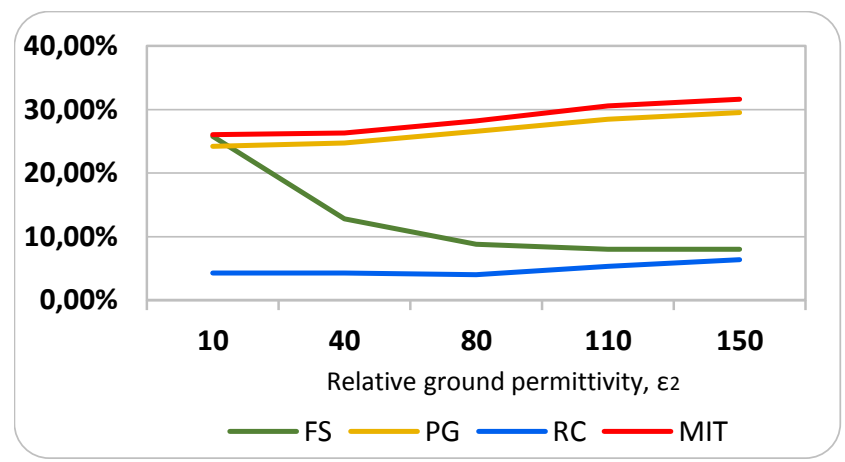

Fig. 8 Percentage of results for the given method with distortion between $25 \%$ and $50 \%$ of NEC results for different $\varepsilon_{2}$ 


\subsection{VARIATIONS OF THE ANTENNA HEIGHT}

The differences in simulated results among the proposed methods for the antenna height variations are presented in Tables 6 and 7, as well in Figures 9 and 10, respectively.

The higher the antenna height above the ground, the lower are differences in the results obtained by NEC and FS simulations. PG and MIT methods again show the worst approximation with the maximum of $36 \%$ of data with distortion up to $25 \%$ of NEC results.

Although differences between the results obtained via RC model and NEC increase with antenna height, $\mathrm{RC}$ method still remains the most accurate approximation.

Table 6 The highest absolute differences between each method and NEC simulation in $\mathrm{mV} / \mathrm{m}$

\begin{tabular}{|c|c|c|c|c|}
\hline \multirow{2}{*}{$\boldsymbol{A}_{\boldsymbol{h}}(\boldsymbol{m})$} & $\boldsymbol{F S}-\boldsymbol{N E C}$ & $\boldsymbol{P G}-\boldsymbol{N E C}$ & $\boldsymbol{R C}-\boldsymbol{N E C}$ & $\boldsymbol{M I T}-\boldsymbol{N E C}$ \\
\hline \multirow{2}{*}{10} & 151,52 & 346,48 & 90,17 & 306,73 \\
\cline { 2 - 5 } & $41,12 \%$ & $153,31 \%$ & $29,59 \%$ & $85,04 \%$ \\
\hline \multirow{2}{*}{20} & 70,99 & 198,07 & 51,42 & 174,25 \\
\cline { 2 - 5 } & $37,02 \%$ & $220,06 \%$ & $53,60 \%$ & $193,21 \%$ \\
\hline \multirow{2}{*}{30} & 47,28 & 137,63 & 34,98 & 121,21 \\
\cline { 2 - 5 } & $86,52 \%$ & $251,55 \%$ & $37,85 \%$ & $221,54 \%$ \\
\hline \multirow{2}{*}{40} & 23,82 & 74,19 & 29,28 & 63,13 \\
\cline { 2 - 5 } & $26,47 \%$ & $107,64 \%$ & $40,74 \%$ & $72,19 \%$ \\
\hline \multirow{2}{*}{50} & 16,40 & 60,20 & 24,85 & 49,80 \\
\cline { 2 - 5 } & $26,04 \%$ & $108,46 \%$ & $43,42 \%$ & $89,73 \%$ \\
\hline
\end{tabular}

Table 7 Percentage of results for the given method within distortion up to $25 \%$ of NEC results

\begin{tabular}{|c|c|c|c|c|}
\hline $\boldsymbol{A}_{\boldsymbol{h}}(\boldsymbol{m})$ & $\boldsymbol{F S}$ - $\boldsymbol{N E C}$ & $\boldsymbol{P G}-\boldsymbol{N E C}$ & $\boldsymbol{R C}-\boldsymbol{N E C}$ & $\boldsymbol{M I T}-\boldsymbol{N E C}$ \\
\hline 10 & $31 \%$ & $14 \%$ & $98 \%$ & $16 \%$ \\
\hline 20 & $62 \%$ & $29 \%$ & $93 \%$ & $32 \%$ \\
\hline 30 & $73 \%$ & $27 \%$ & $89 \%$ & $29 \%$ \\
\hline 40 & $81 \%$ & $34 \%$ & $88 \%$ & $39 \%$ \\
\hline 50 & $86 \%$ & $27 \%$ & $85 \%$ & $38 \%$ \\
\hline
\end{tabular}

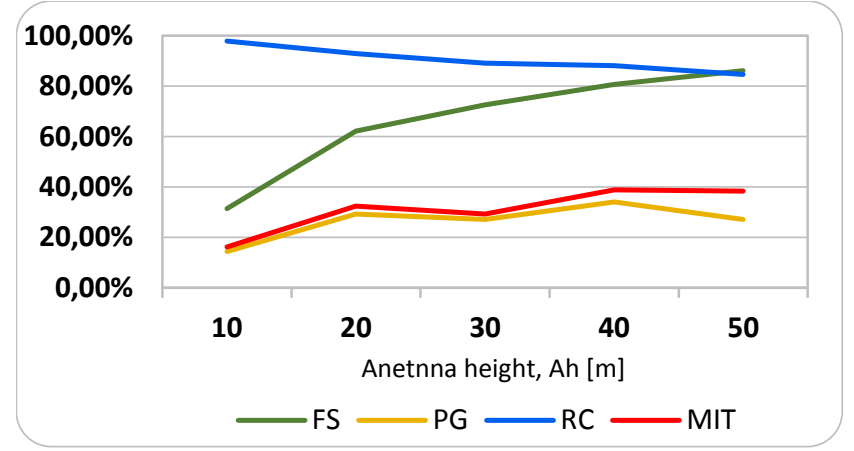

Fig. 9 Percentage of results for the given method within distortion up to 25\% of NEC results for different $A_{h}$ 


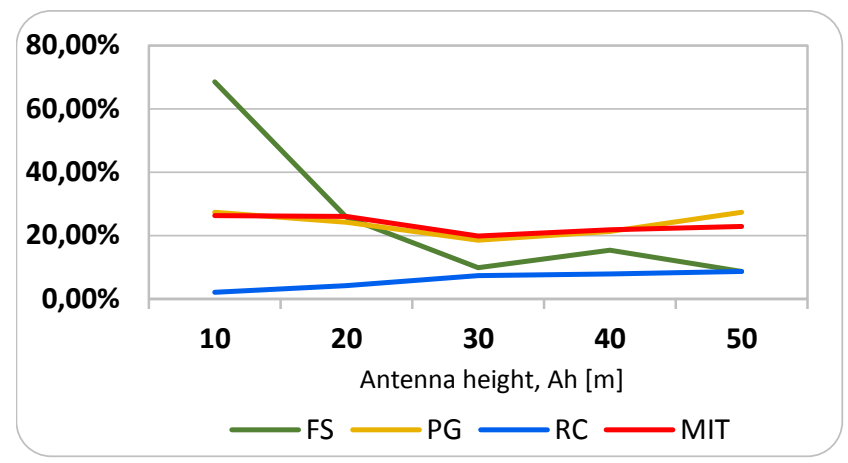

Fig. 10 Percentage of results for the given method with distortion between $25 \%$ and $50 \%$ of NEC results for different $A_{h}$

\subsection{RADIATED FIELD VS. DISTANCE}

According to the presented results, it can be seen that $\mathrm{RC}$ approach shows the smallest distortions compared to NEC simulation (Figures 11 and 12). The PG and MIT methods provide similar results with a satisfactory approximation only at high ground conductance values (Figure 13). For all the other values and all the other calculations these two methods show the worst approximation of all. FS method provides acceptable approximation for higher relative permittivity values and for higher Ah values (above $30 \mathrm{~m}$ ). For those specific cases FS method is similar to RC model and NEC, respectively (Figure 14).

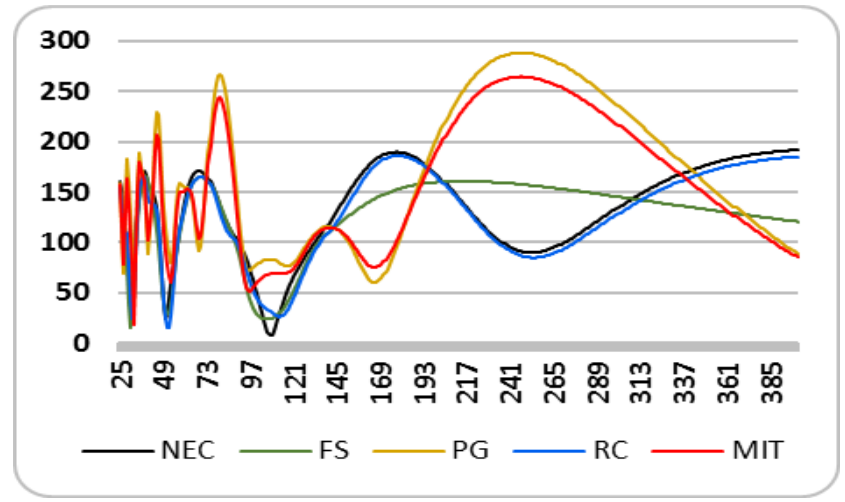

Fig. 11 Electric field computed via different approaches compared to NEC simulation in $\mathrm{mV} / \mathrm{m}$ at specific distance from antenna $\left(\sigma_{2}=1000 \mathrm{~S} / \mathrm{m}, \varepsilon_{2}=110\right.$ and $\left.A_{h}=40 \mathrm{~m}\right)$ 




Fig. 12 Differences in electric field strength between the given method and NEC simulation in $\mathrm{mV} / \mathrm{m}$ at specific distance from antenna $\left(\sigma_{2}=0.01 \mathrm{~S} / \mathrm{m}, \varepsilon_{2}=10\right.$ and $\left.A_{h}=20 \mathrm{~m}\right)$

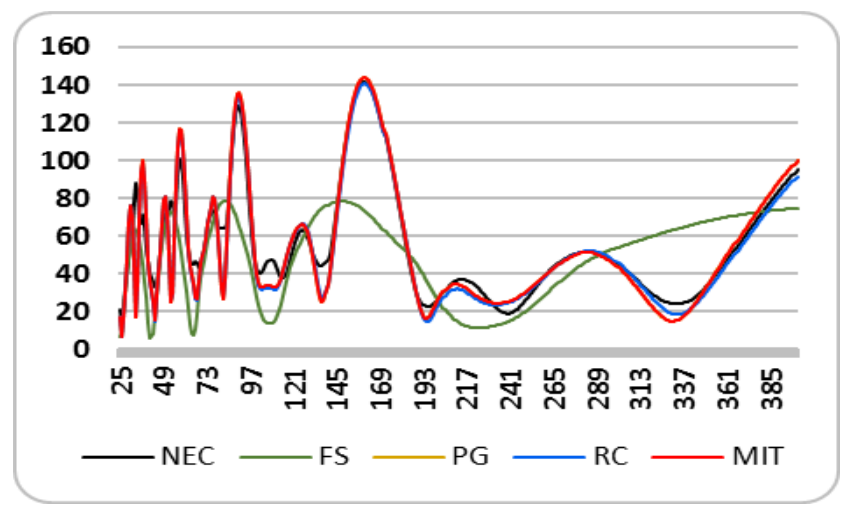

Fig. 13 Electric field computed via different approaches compared to NEC simulation in $\mathrm{mV} / \mathrm{m}$ at specific distance from antenna ( $\sigma=1000 \mathrm{~S} / \mathrm{m}, \varepsilon 2=110$ and $\mathrm{Ah}=40 \mathrm{~m})$

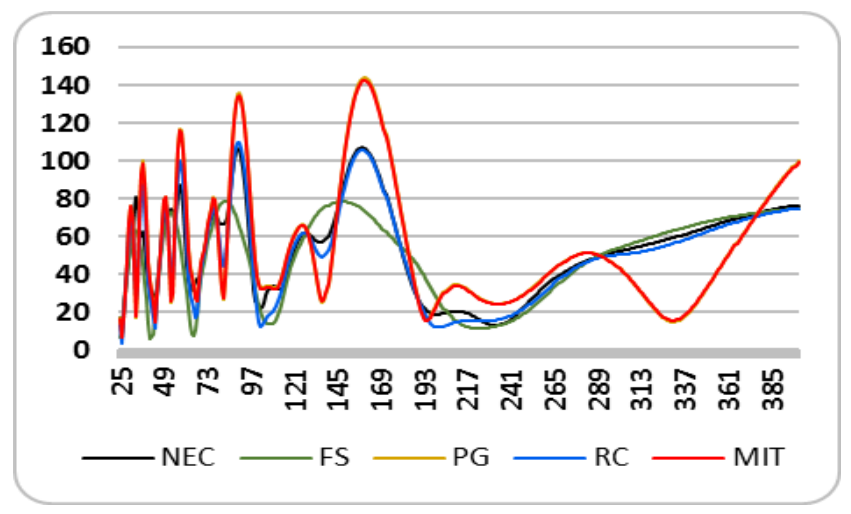

Fig. 14 Electric field computed via different approaches compared to NEC simulation in $\mathrm{mV} / \mathrm{m}$ at specific distance from antenna $\left(\sigma=0.01 \mathrm{~S} / \mathrm{m}, \varepsilon_{2}=110\right.$ and $\left.A_{h}=40 \mathrm{~m}\right)$ 


\section{CONCLUSION}

The paper deals with four different models for the assessment of the electric field radiated by the base station antenna system: the antenna insulated in Free Space (FS), the antenna above Perfect Ground (PG) and the antenna above a lossy half space. The half-space is taken into account via Fresnel Reflection Coefficient (RC) and Modified Image Theory Method (MIT), respectively. The results obtained by these models are compared to the results obtained by NEC as benchmark.

The PG and MIT methods are considered to be satisfactory approximations only for high ground conductivity values and provide similar results. The FS method provides a good approximation for higher relative permittivity values (above 10) and for higher Ah values as well. Finally, RC approach provides rather satisfactory results in all cases.

\section{APPENDIX A}

The power flux $P$ (radiated power of an antenna) is given by integral:

$$
\oint \vec{E} \times \vec{H} d \vec{S}=\oint S d \vec{S}
$$

where product $\vec{E} \times \vec{H}$ presents the power density, i.e. Poynting vector $S$.

Poynting vector in the free space for root-mean-square values can be written, as follows:

$$
S=\frac{E^{2}}{Z_{0}}=\frac{E^{2}}{120 \pi}
$$

Combining (A.1) and (A.2) for the case of an isotropic radiator yields:

$$
S=\frac{E^{2}}{120 \pi}=\frac{P}{4 \pi * r^{2}}
$$

and the electric field strength is given by:

$$
E=\frac{\sqrt{30 * P}}{r}
$$

Taking into account the number of antenna channels $N$, as well as horizontal and vertical antenna gain $G$ follows:

$$
E=\frac{\sqrt{30 * N * P * 10^{\frac{G}{10}}}}{r}
$$

Note that expression (A.5) is related to directional radiators.

\section{REFERENCES}

[1] R.W.Y. Habash, Electromagnetic Fields and Radiation. Marcel, Dekker, New York, 2002.

[2] International Commision on Non-Ionizing Radiation Protection (ICNIRP), Guidelines for Limiting Exposure to Time-Varying, Electric, Magnetic and Electromagnetic Fields (up to 300 GHz). Health Phys., Vol. 74, No. 4, pp. 494-522, 1998. 
[3] M. Galić, D. Poljak and V. Dorić, Comparison of Different Analytical Models to Determine Electric Field Radiated by a Base Station Antenna, SpliTECH 2017, Split, 2017.

[4] M. Galić, D. Poljak and V. Dorić, Comparison of Free Space, Perfect Ground and Fresnel's Equation Models to Determine Electric Field Radiated by a Base Station Antenna, SoftCOM 2017, Split 2017. DOI: 10.23919/softcom.2017.8115517

[5] P. Bernardi, M. Cavagnaro, S. Pisa and E. Piuzzi, Human exposure to radio base station antennas in urban enviroment, IEEE Trans. MTT, Vol. 48, No. 11, pp. 1996-2002, 2000.

DOI: $\underline{10.1109 / 22.884188}$

[6] P. Bernardi, M. Cavagnaro, S. Pisa and E. Piuzzi, Human exposure in the vicinity of radio base station antennas, 4th European Symposium on Electromagnetic Compatibility (EMCEurope 2000), Brugge, Belgium, pp. 187-192, Sept. 11-15, 2000.

[7] V. Labinac and B. Milotić, Waves and Optics (Valovi i optika, page 6), University of Rijeka, 2015.

[8] D. Poljak, Electromagnetic Field Theory With Engineering Applications (in Croatian), Školska knjiga Zagreb, Zagreb 2014.

[9] T. Takashima, T. Nakae and R. Ishibashi, Calculation of complex fields in conducting media, IEEE Transactions on Electrical Insulation, Vol. EI-15, No. 1, pp. 1-7, 1980.

DOI: $10.1109 /$ tei.1980.298290

[10] D. Poljak, V. Dorić, M. Birkić, K. El Khamlichi Drissi, S. Lallechere and L. Pajewski, A simple analysis of dipole antenna radiation above a multilayered medium, IWAGPR2017, Edinburgh, 2017. DOI: 10.1109/iwagpr.2017.7996037

[11] 4nec2, www.qsl.net/4nec2, July 2017.

[12] European Standard EN 61566, Measurement of exposure to radio-frequency electromagnetic fields Field strength in the frequency range $100 \mathrm{kHz}$ to $1 \mathrm{GHz}$ (IEC 61566:1997), 1997. DOI: $10.3403 / 01148902$

[13] D. Poljak, Human Exposure to the Radiation of Base Station Antenna systems - Incident Field Dosimetry, ZIRS, 2012 (in Croatian). 